Supporting Information

\title{
Oxanosine is a Substrate of Adenosine Deaminase. Implications for the Quest for a Toxicological Marker for Nitrosation Activity
}

\author{
Papiya Majumdar, Hong Wu, Peter Tipton, and Rainer Glaser \\ Departments of Chemistry and Biochemistry, University of Missouri-Columbia, Columbia, \\ Missouri 65211
}

Table of Contents

Figure S1: Monitoring the ADA-cat. hydrolysis by UV/Vis spectroscopy S2

Figure S2: ${ }^{1} \mathrm{H}$ NMR spectrum of $4 r$ in ${\text { DMSO }-d_{6} .}$ S3

Figure S3. Conformers and Isomers of 4h. S4

Figure S4. Conformer of $4 \mathrm{~m}$.

Table S1: Total Energies and Thermodynamical Data at B3LYP/6-311G** . ${ }^{a}$ S6

Table S2: Measured and Calculated ${ }^{13} \mathrm{C}$ Chem. Shifts of $3 \mathrm{~h}, 4 \mathrm{~h}$, and $4 \mathrm{~m}^{\mathrm{a}} \mathrm{S} 7$

Table s3: Calculated Chemical Shifts ${ }^{a}\left(\mathrm{ppm}\right.$ relative to TMS $\left.^{b}\right) . \quad$ S8

Cartesian Coordinates of Optimized Structures S10

$\begin{array}{ll}\text { Total Pages } & 13\end{array}$ 


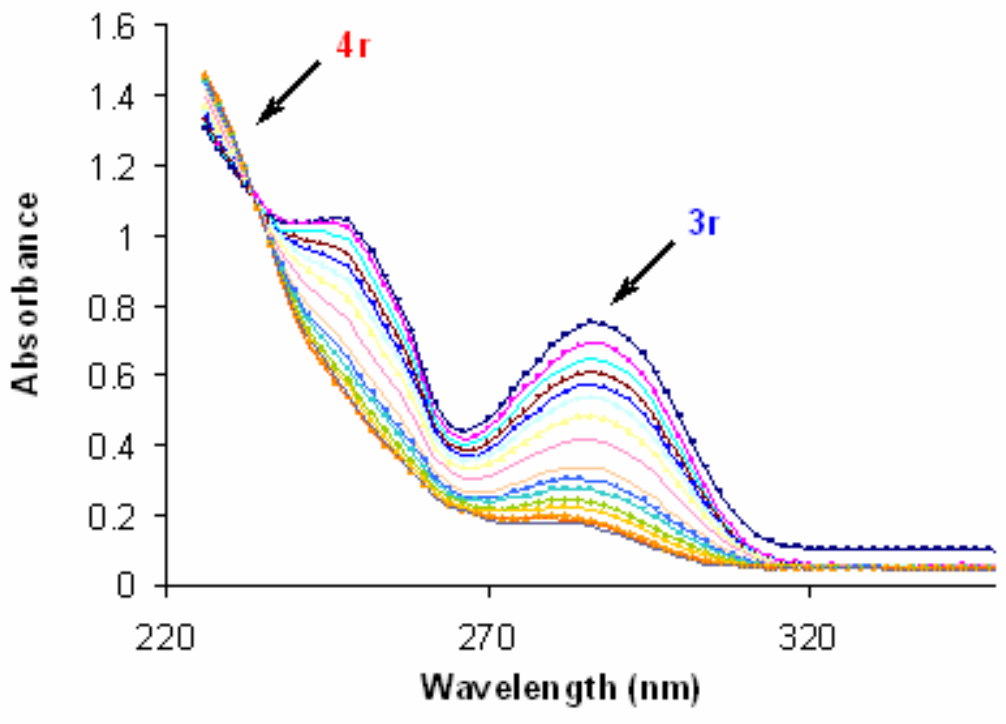

Figure S1: Monitoring the ADA-catalyzed hydrolysis by UV/Vis spectroscopy. UV spectra were measured at one-second intervals for the ADA-catalyzed hydrolysis of $0.01 \mathrm{mM} \mathrm{3r}$. The reaction was complete after 40 minutes and some data are omitted for clarity. 


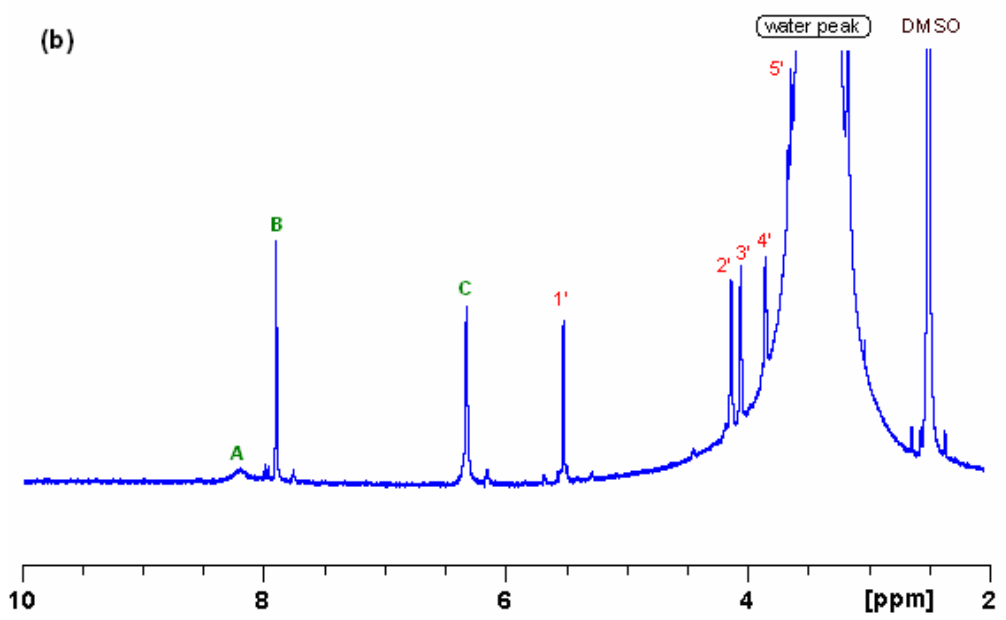

Figure S2: ${ }^{1} \mathrm{H}$ NMR spectrum of $4 \mathbf{r}$ in DMSO- $\mathrm{d}_{6}$. 
Figure S3. Conformers and Isomers of $\mathbf{4 h}$ and their relative energies to $4 \mathbf{h}\left(\mathrm{kcal} \mathrm{mol}^{-1}\right)$

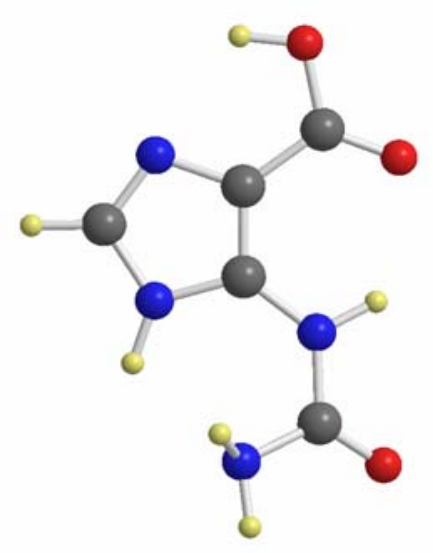

4h-conformer1

8.35

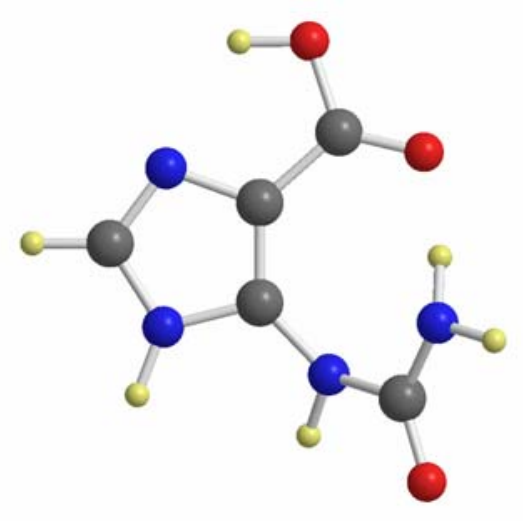

4h-conformer2

9.49

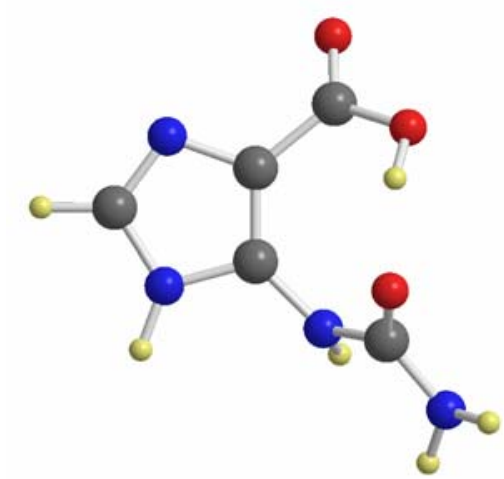

4h-conformer3

19.18

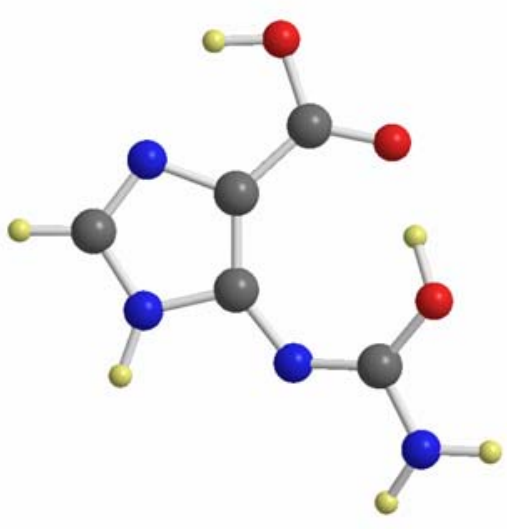

4h-isomer1

10.56

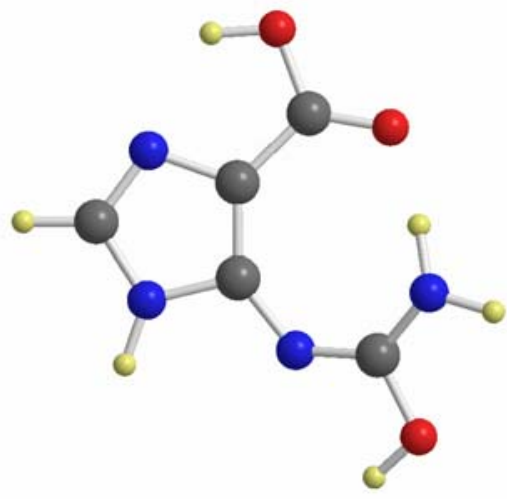

4h-isomer2

14.10 
Figure S4. Conformer of $\mathbf{4 m}$ and its relative energy to $\mathbf{4 m}\left(\mathrm{kcal} \mathrm{mol}^{-1}\right)$

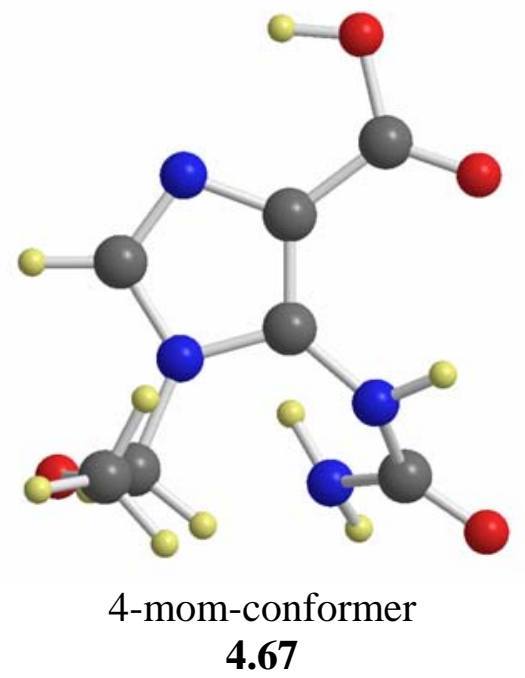


Table S1. Total Energies and Thermodynamical Data at B3LYP/6-311G**. ${ }^{a}$

\begin{tabular}{ccccccc}
\hline Molecule & Sym & $\boldsymbol{E}$ & $\boldsymbol{V Z P E}$ & $\boldsymbol{T E}$ & $\boldsymbol{S}$ & $\boldsymbol{N}$ \\
\hline oxanosine & $C_{1}$ & -562.560352 & 64.63 & 69.89 & 89.67 & 0 \\
4h & $C_{1}$ & -639.060151 & 82.12 & 88.75 & 101.42 & 0 \\
4h-conformer1 & $C_{1}$ & -639.046848 & 82.42 & 88.92 & 100.98 & 0 \\
4h- conformer2 & $C_{1}$ & -639.045021 & 82.46 & 88.85 & 99.95 & 0 \\
4h- conformer3 & $C_{1}$ & -639.029579 & 81.72 & 88.39 & 101.53 & 0 \\
4h-isomer1 & $C_{1}$ & -639.043329 & 81.68 & 88.06 & 99.64 & 0 \\
4h-isomer2 & $C_{1}$ & -639.037681 & 82.37 & 88.65 & 98.96 & 0 \\
4m & $C_{1}$ & -792.919601 & 120.31 & 129.55 & 123.66 & 0 \\
4m-conformer & $C_{1}$ & -792.912163 & 120.31 & 129.49 & 123.85 & 0
\end{tabular}

${ }^{a}$ Total energies $E$ in atomic units. Vibrational zero-point energies VZPE and thermal energies TE in $\mathrm{kcal} \mathrm{mol}^{-1}$. Molecular entropies $S$ in cal $\mathrm{mol}^{-1} \cdot \mathrm{K}^{-1}$. 
Table S2: Measured and Calculated ${ }^{13} \mathrm{C}$ Chemical Shifts of $\mathbf{3 h}, \mathbf{4 h}$, and $\mathbf{4} \mathbf{m}^{a}$

\begin{tabular}{|c|c|c|c|c|c|c|}
\hline Mol. & Method & $C(0)-0$ & $\mathrm{C}(\mathbf{O})-\mathrm{N}$ & C-NH & C2 & $\mathrm{C}-\mathrm{CO}_{2}$ \\
\hline & & C7 & C5 & CЗа & C2 & C7a \\
\hline \multirow[t]{5}{*}{$3 \mathbf{h}$} & A & 154.7 & 165.8 & 157.5 & 135.2 & 117.2 \\
\hline & B & 156.3 & 166.4 & 158.1 & 135.1 & 117.7 \\
\hline & C & 159.2 & 169.7 & 162.0 & 138.8 & 120.6 \\
\hline & Exptl. & 161.8 & 155.8 & 154.3 & 138.8 & 112.7 \\
\hline & & C4a & C5a & C5 & $\mathrm{C} 2$ & C4 \\
\hline \multirow[t]{3}{*}{$4 h$} & A & 168.3 & 156.6 & 143.0 & 130.1 & 119.0 \\
\hline & B & 169.9 & 157.6 & 142.8 & 129.9 & 119.4 \\
\hline & C & 173.3 & 160.8 & 145.8 & 133.5 & 122.5 \\
\hline \multirow[t]{3}{*}{$4 m$} & A & 168.7 & 158.5 & 143.1 & 135.4 & 122.8 \\
\hline & B & 170.4 & 159.3 & 142.8 & 134.8 & 122.8 \\
\hline & C & 173.8 & 162.3 & 145.9 & 138.6 & 126.0 \\
\hline $4 h$ & A & 170.2 & 159.3 & 146.2 & 134.6 & 117.7 \\
\hline \multirow[t]{2}{*}{$(\mathrm{MeOH})$} & B & 171.9 & 160.5 & 146.3 & 134.3 & 117.7 \\
\hline & C & 175.3 & 163.9 & 149.4 & 138.2 & 120.9 \\
\hline 4-mom & A & 170.6 & 161.2 & 145.8 & 138.6 & 122.3 \\
\hline \multirow[t]{3}{*}{$(\mathrm{MeOH})$} & B & 172.4 & 162.1 & 145.5 & 139.0 & 122.0 \\
\hline & $\mathrm{C}$ & 175.8 & 165.3 & 148.8 & 142.0 & 125.3 \\
\hline & Exptl. & 167.7 & 160.2 & 132.2 & 134.1 & 128.3 \\
\hline
\end{tabular}

${ }^{a}$ The basis set is given to describe the theoretical method, see text.

${ }^{b}$ The shielding data computed for tetramethylsilane (TMS) are used as reference; in gas phase 184.7 at B3LYP/A, 183.9 at B3LYP/B//B3LYP/A, and 181.6 at B3LYP/C//B3LYP/A, and in methanol 185.2 at B3LYP/A, 184.5 at B3LYP/B//B3LYP/A, and 182.2 at B3LYP/C//B3LYP/A. 
Table S3. Calculated Chemical Shifts ${ }^{a}$ (ppm relative to $\mathrm{TMS}^{b}$ )

\begin{tabular}{|c|c|c|c|c|c|c|}
\hline Molecule & Basis Set & C7 & C5 & C3a & C2 & C7a \\
\hline \multirow[t]{3}{*}{ oxanosine } & A & 154.7 & 165.8 & 157.5 & 135.2 & 117.2 \\
\hline & B & 156.3 & 166.4 & 158.1 & 135.1 & 117.7 \\
\hline & $\mathrm{C}$ & 159.2 & 169.7 & 162.0 & 138.8 & 120.6 \\
\hline \multirow[t]{3}{*}{$4 h$} & A & 168.3 & 156.6 & 143.0 & 130.1 & 119.0 \\
\hline & B & 169.9 & 157.6 & 142.8 & 129.9 & 119.4 \\
\hline & $\mathrm{C}$ & 173.3 & 160.8 & 145.8 & 133.5 & 122.5 \\
\hline \multirow[t]{3}{*}{ 4h-conformer 1} & A & 166.4 & 155.7 & 140.6 & 129.6 & 119.9 \\
\hline & B & 168.1 & 156.9 & 140.2 & 129.3 & 120.2 \\
\hline & C & 171.4 & 160.0 & 143.5 & 132.9 & 123.4 \\
\hline \multirow[t]{3}{*}{ 4h-conformer2 } & A & 162.8 & 160.9 & 127.6 & 135.7 & 139.9 \\
\hline & B & 169.3 & 160.5 & 139.2 & 130.8 & 139.1 \\
\hline & $\mathrm{C}$ & 172.4 & 164.0 & 142.2 & 134.4 & 130.6 \\
\hline \multirow[t]{3}{*}{ 4h-conformer3 } & A & 163.3 & 161.7 & 128.3 & 135.2 & 141.4 \\
\hline & B & 165.0 & 162.4 & 127.3 & 134.8 & 142.2 \\
\hline & C & 168.0 & 165.4 & 130.0 & 138.2 & 146.2 \\
\hline \multirow[t]{3}{*}{ 4h-isomer1 } & A & 173.0 & 162.3 & 148.6 & 128.7 & 119.8 \\
\hline & B & 174.2 & 163.0 & 149.0 & 130.2 & 120.0 \\
\hline & $\mathrm{C}$ & 177.6 & 166.3 & 152.6 & 134.0 & 123.4 \\
\hline \multirow[t]{3}{*}{ 4h-isomer2 } & A & 170.1 & 161.2 & 145.7 & 130.4 & 122.7 \\
\hline & B & 171.4 & 161.6 & 146.0 & 129.9 & 122.6 \\
\hline & C & 174.6 & 164.8 & 149.6 & 133.6 & 126.2 \\
\hline \multirow[t]{3}{*}{ 4h-mom } & A & 168.7 & 158.5 & 143.1 & 135.4 & 122.8 \\
\hline & B & 170.4 & 159.3 & 142.8 & 134.8 & 122.8 \\
\hline & C & 173.8 & 162.3 & 145.9 & 138.6 & 126.0 \\
\hline
\end{tabular}




$\begin{array}{lllllll}\text { 4h-mom1 } & \text { A } & 165.1 & 158.1 & 139.7 & 136.8 & 127.7 \\ & \text { B } & 166.9 & 159.4 & 139.5 & 136.2 & 127.4 \\ & \text { C } & 170.2 & 162.5 & 142.9 & 139.9 & 130.7\end{array}$

$\begin{array}{lllllll}\text { 4h (in DMSO) } & \text { A } & 170.2 & 159.3 & 146.1 & 134.7 & 117.7 \\ & \text { B } & 172.0 & 160.5 & 146.2 & 134.4 & 117.7 \\ & \text { C } & 175.3 & 163.9 & 149.4 & 138.3 & 120.9\end{array}$

$\begin{array}{lllllll}\text { 4h }\left(\text { in } \mathrm{CH}_{3} \mathrm{OH}\right) & \text { A } & 170.2 & 159.3 & 146.2 & 134.6 & 117.7\end{array}$

$\begin{array}{llllll}\text { B } & 171.9 & 160.5 & 146.3 & 134.3 & 117.7\end{array}$

$\begin{array}{llllll}\text { C } & 175.3 & 163.9 & 149.4 & 138.2 & 120.9\end{array}$

$\begin{array}{lllllll}\text { 4m (in DMSO) } & \text { A } & 170.6 & 161.1 & 145.7 & 138.7 & 122.3 \\ & \text { B } & 172.4 & 162.1 & 145.5 & 138.1 & 122.0 \\ & \text { C } & 175.8 & 165.2 & 148.7 & 142.1 & 125.3\end{array}$

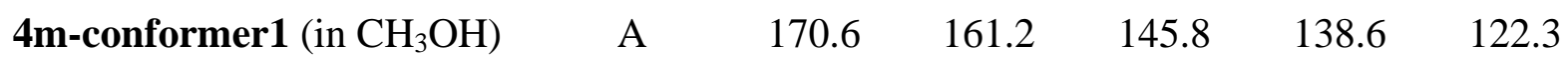

$\begin{array}{llllll}\text { B } & 172.4 & 162.1 & 145.5 & 139.0 & 122.0\end{array}$

$\begin{array}{llllll}\text { C } & 175.8 & 165.3 & 148.8 & 142.0 & 125.3\end{array}$

${ }^{a}$ NMR calculations are carried out based on the structures optimized at B3LYP/6-3llG**. Basis set $A$ is 6-311G**. Basis set $B$ is cc-pVTZ. Basis set $C$ is cc-pVQZ.

${ }^{b}$ The calculated shielding constant of TMS: 184.7 at B3LYP/6-311G**// B3LYP/6-311G**, 183.9 at B3LYP/cc-pVTZ// B3LYP/6-311G**, and 181.6 at B3LYP/cc-pVQZ// B3LYP/6$311 \mathrm{G}^{* *}$. 


\section{Cartesian Coordinates of Optimized Structures}

\author{
Oxanosine \\ 01 \\ O $\quad-1.6413637903$ \\ C -1.5083593023 \\ $\mathrm{N} \quad-0.3861269145$ \\ C $\quad 0.6876388785$ \\ C $\quad 0.7216051181$ \\ C $\quad-0.5001801608$ \\ $\mathrm{N} \quad 1.9963620181$ \\ C $\quad 2.7543815004$ \\ $\mathrm{N} \quad 2.0234935723$ \\ O $\quad-0.78453158$ \\ $\mathrm{N} \quad-2.695653569$ \\ $\mathrm{H} \quad 2.3244881163$ \\ $\mathrm{H} \quad 3.8323460889$ \\ $\mathrm{H} \quad-2.7040900133$ \\ $\mathrm{H} \quad-3.5426231812$
}

$4 \mathrm{~h}$

$\begin{array}{ll}0 & 1 \\ \mathrm{O} & -0.5056958855 \\ \mathrm{~N} & -0.7292808051 \\ \mathrm{C} & 0.3691025627 \\ \mathrm{C} & 0.3980384787 \\ \mathrm{C} & -0.7680146581 \\ \mathrm{~N} & 1.6699498706 \\ \mathrm{C} & 2.441016288 \\ \mathrm{~N} & 1.7060073702 \\ \mathrm{O} & -1.9066206587 \\ \mathrm{C} & -0.6515787474 \\ \mathrm{~N} & -1.8617844781 \\ \mathrm{O} & 0.4161127524 \\ \mathrm{H} & 0.459689808 \\ \mathrm{H} & -1.6039080736 \\ \mathrm{H} & 1.9347646082 \\ \mathrm{H} & 3.517640237 \\ \mathrm{H} & -1.8423882934 \\ \mathrm{H} & -2.7217951983\end{array}$

$4 m$

01

$\begin{array}{ll}\mathrm{O} & -1.531861305 \\ \mathrm{~N} & -1.1794076044 \\ \mathrm{C} & -0.1734586235 \\ \mathrm{C} & -0.3466017062 \\ \mathrm{C} & -1.6236098932 \\ \mathrm{~N} & 1.1821877285 \\ \mathrm{C} & 1.7628899225 \\ \mathrm{~N} & 0.8747733623 \\ \mathrm{O} & -2.6987932933 \\ \mathrm{C} & -1.2283810794 \\ \mathrm{~N} & -2.462705458 \\ \mathrm{O} & -0.254475548 \\ \mathrm{C} & 1.9443286893 \\ \mathrm{O} & 2.9949418347 \\ \mathrm{C} & 2.5920443693 \\ \mathrm{H} & -0.5859896467 \\ \mathrm{H} & -2.0643057433 \\ \mathrm{H} & 2.8342918262 \\ \mathrm{H} & -2.5713346479 \\ \mathrm{H} & -3.2933424875\end{array}$

0.4480797602

$-0.8218599916$

$-1.4730759823$

$-0.7029224496$

0.6230461561

1.3366422266

$-1.085372849$

0.0125198367

1.0480991399

2. 4372590028

$-1.4049479423$

$-1.9974591442$

$-0.0294941391$

$-2.3951551866$

$-0.9380728679$

3. 3481593455

$-0.981117893$

$-0.1580626937$

1. 2026958184

2.0474846818

$-0.5408223225$

0.5920301829

1.6485136788

1. 616305142

$-2.3439933578$

$-2.9808964111$

$-2.924777774$

3. 4586342294

$-0.4873002933$

$-1.5029974034$

0.5620541887

$-3.9375485456$

$-2.4710030384$

3.5211764071

$-0.8240236096$

0.1251797532

1. 4891863382

2. 1927713232

$-0.07376414$

1.1734678249

2.125736259

1.6339415358

$-1.9918279069$

$-2.584616205$

$-2.4619131489$

- 1.3212100678

$-1.1046065993$

$-0.9824856598$

3. 7415945305

$-0.343554778$

1. 2917669857

$-3.3512621633$

$-2.0760215817$

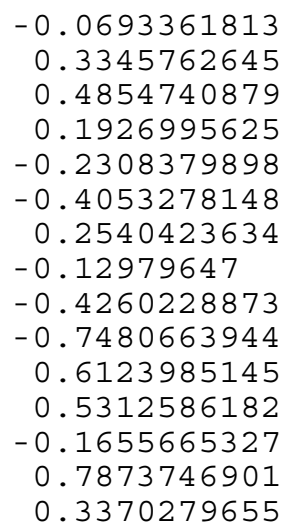

0.6186198953

$-0.1469724933$

$-0.04743601$

0.1943681813

0.4001056472

$-0.1739625773$

$-0.0058867723$

0.2153698463

0.3782047439

$-0.3845299495$

$-0.4152930991$

$-0.5251496501$

0.5930609881

0.01196067

$-0.3499620368$

$-0.0591501167$

$-0.727932851$

$-0.535094883$

0.4490916572

$-0.0541209707$

0.0794636924

0.2210863723

0.270460607

0.0261305509

0.1654567139

0.2708165472

0.1528720729

0.7240829606

0.7314296166

1. 2803117379

$-0.2023427705$

$-1.0933014335$

$-2.4553916664$

0.5037218819

$-0.2121282303$

0.1628219679

1.3747968409

0.4746133708 


$\begin{array}{ll}\mathrm{H} & 1.2390214115 \\ \mathrm{H} & 2.380879871 \\ \mathrm{H} & 1.9800218173 \\ \mathrm{H} & 3.5051360738 \\ \mathrm{H} & 2.0299217465\end{array}$

$-2.0758321215$

$-1.6509577229$

$-0.08832073$

$-0.902146102$

$-1.8678676343$

4h-conformer 1

01

O $\quad-0.9372400191$

$\mathrm{N} \quad-0.5964902059$

C $\quad 0.348954845$

C $\quad 0.1946110503$

C $\quad-1.0373594129$

$\mathrm{N} \quad 1.6737389107$

C 2.2652937493

$\mathrm{N} \quad 1.4025664132$

O $\quad-2.0753120047$

C $\quad-0.4972184184$

$\mathrm{N} \quad 0.5732570346$

O $\quad-1.256933277$

$\mathrm{H} \quad-0.0191570506$

$\mathrm{H} \quad-1.4577613352$

$\mathrm{H} \quad 2.1136462345$

$\mathrm{H} \quad 3.3134415103$

$\mathrm{H} \quad 0.544870089$

$\mathrm{H} \quad 0.6436470101$

\section{4h-conformer 2}

01

O $\quad 0.2180705724$

$\mathrm{N} \quad-0.2532509519$

C $\quad 0.6659303096$

C $\quad 0.7135537154$

C $\quad-0.2685646396$

$\mathrm{N} \quad 1.9162047518$

C $\quad 2.6531099921$

$\mathrm{N} \quad 1.9560178902$

O $\quad-1.4189138177$

C -1.5466027659

$\mathrm{N} \quad-2.2065180628$

O -1.9432150498

$\mathrm{H} \quad 1.1429935102$

$\mathrm{H} \quad 0.1423316935$

$\mathrm{H} \quad 2.2022068804$

$\mathrm{H} \quad 3.676224696$

$\mathrm{H} \quad-3.1532139767$

$\mathrm{H} \quad-2.0498115039$

\section{4h-conformer 3}

01

O $\quad-1.5607982937$

$\mathrm{N} \quad-0.2665789497$

C $\quad 0.7205829502$

C $\quad 0.720973248$

C $\quad-0.4239699064$

N $\quad 2.0497165917$

C $\quad 2.7773058075$

$\mathrm{N} \quad 2.0113033916$

O $\quad-0.3445134189$

C $\quad-1.2771487786$

$\mathrm{N} \quad-2.1270841873$

O -1.4124416029

$\mathrm{H} \quad-1.4221569276$

$\mathrm{H} \quad-0.4262943552$

3.2233140314

2. 7058772033

$-1.5960095539$

$-0.5593775118$

0.7409303296

1. 5419152975

$-0.7611070954$

0.3818675473

1.2975818899

1.2157264969

$-1.665520182$

$-0.4841023363$

$-2.7446500903$

2.7695325541

$-2.526024896$

$-1.5928384778$

0.4693563629

$-0.5807307966$

0.2116431608
1.463840544

$-1.3862552268$

$-0.4248332808$

0.941071224

1.9063175392

$-0.7976729906$

0.3333161349

1.3894689079

3.0006872597

$-1.6980201638$

$-2.6809754945$

$-1.1215414753$

0.6234923548

$-1.6207462023$

-0.5516780254
0.7382408026
-2.6185108523
-3.0433597632
-2.7769959327

$-0.7955865422$

0.5242467673

$-0.0117219536$

$-0.4086979001$

$-0.3375340862$

$-0.214437617$

$-0.7177702583$

$-0.8445270522$

0.1057943192

0.8483709262

0.227821947

1.6208446406

$-1.083098167$

0.79420077

0.0425030734

$-0.9693957258$

0.451296143

$-0.7715341185$

1.5267832534

$-0.1363801128$

$-0.1541076853$

0.3299732941

1. 075901163

$-0.7050195547$

$-0.5349589181$

0.0748434703

1.2866537709

$-0.7368725678$

$-0.8284439955$

$-1.1269291508$

1. 2255281148

$-0.1070659949$

$-1.1999117749$

$-0.8658229353$

$-1.162739494$

$-0.0966612691$

$-0.4613230761$

0.6038335884

0.3258932379

0.115207638

0.1075395038

0.2842981726

0.056340827

$-0.0452448935$

0.5939449367

$-0.299204433$

0.1304233903

$-1.3679936054$

$-0.9468451836$

1. 5744287655 
$\mathrm{H}$

\section{4h-isomer 1 \\ 01 \\ 6693023644 \\ $\mathrm{N} \quad-0.4129580516$ \\ C $\quad 0.693615027$ \\ C $\quad 0.6883088219$ \\ C $\quad-0.544933136$ \\ $\mathrm{N} \quad 1.9885827573$ \\ C $\quad 2.7221197746$ \\ $\mathrm{N} \quad 1.9719032737$ \\ O $\quad-0.7819475957$ \\ $\mathrm{N} \quad-2.6882731684$ \\ $\mathrm{H} \quad-0.4097601657$ \\ $\mathrm{H} \quad 2.350456512$ \\ $\mathrm{H} \quad 3.7904284068$ \\ $\mathrm{H} \quad-3.5181630836$}

\section{4h-isomer 2}

$\begin{array}{lc}\mathrm{O} & 1 \\ \mathrm{O} & 0.2274917557 \\ \mathrm{~N} & -0.1978534384 \\ \mathrm{C} & 0.675703033 \\ \mathrm{C} & 0.7274964412 \\ \mathrm{C} & -0.2849493814 \\ \mathrm{~N} & 2.0068609221 \\ \mathrm{C} & 2.7815582424 \\ \mathrm{~N} & 2.0457793919 \\ \mathrm{O} & -1.4903145527 \\ \mathrm{C} & -1.4499532077 \\ \mathrm{~N} & -2.2373658289 \\ \mathrm{O} & -2.07860961 \\ \mathrm{H} & 1.1958580914 \\ \mathrm{H} & 2.3042479998 \\ \mathrm{H} & 3.8567748589 \\ \mathrm{H} & -3.2088095248 \\ \mathrm{H} & -2.0316882886 \\ \mathrm{H} & -1.4060019733\end{array}$

\section{4m-conformer}

01

$\begin{array}{lr}\mathrm{O} & -0.7966765528 \\ \mathrm{~N} & -1.2543865368 \\ \mathrm{C} & -0.1496564832 \\ \mathrm{C} & -0.0655069365 \\ \mathrm{C} & -1.1781030096 \\ \mathrm{~N} & 1.1597134652 \\ \mathrm{C} & 1.9570131264 \\ \mathrm{~N} & 1.2537347613 \\ \mathrm{O} & -2.3367330004 \\ \mathrm{C} & -1.6630368146 \\ \mathrm{~N} & -0.7614263562 \\ \mathrm{O} & -2.6969175523 \\ \mathrm{C} & 1.6423210752 \\ \mathrm{O} & 2.5587511557 \\ \mathrm{C} & 1.9795784434 \\ \mathrm{H} & 0.1742075488 \\ \mathrm{H} & -2.0496145125 \\ \mathrm{H} & 3.0333201544 \\ \mathrm{H} & -1.1385669478 \\ & \end{array}$
$-1.7413064001$

0.3242376907

$-3.3742555607$

0.4807863123

$-0.8832364817$

$-1.4966482795$

$-0.7289481497$

0.6504166985

1.3750834018

$-1.1103890289$

0.0715014428

1.1273730748

2.5394861786

$-1.5985547393$

$-2.5038340774$

$-2.0512139452$

0.0507599832

$-1.0192605468$

3.0106048401

$-1.6567860848$

$-0.6113329779$

0.7790066928

1. 7942885326

$-0.9566032265$

0.1635155258

1. 2169246786

1.6151743959

$-1.6469079959$

$-0.6367091516$

$-2.8421257436$

2. 9287597424

$-1.9082389168$

0.1431930455

$-0.8711064254$

0.2974952822

$-3.4985328409$

3.4723526693

$-0.6413198195$

0.1153063346

1.3907213943

2. 3006644864

$-0.2964658197$

0.7361287112

1. 7596470796

2.0343701029

$-1.8517671042$

$-2.3907316542$

$-2.3880337077$

$-1.5262137089$

$-1.2470417049$

$-0.700508594$

3. 468340831

$-0.0862000701$

0.669299188

$-3.1862151109$
0.3828966858

$-0.0237428767$

0.7922658118

0.2151440313

0.2669054621

0.1157273979

$-0.0689342077$

$-0.1215377975$

0.0231298862

$-0.2355310385$

$-0.3839641165$

$-0.3197385429$

0.0112935754

0.4382822051

0.1542301801

$-0.249821782$

$-0.5327671148$

0.532082353

0.7018188948

$-0.0527543683$

$-0.0261100766$

0.1716475686

0.4758829786

$-0.1749047992$

$-0.0763915037$

0.1331660938

0.5373864149

$-0.3892341676$

$-0.7843561202$

$-0.4117317976$

0.6007137655

$-0.3272025039$

$-0.1640542569$

$-0.9226444361$

$-0.4027661778$

$-0.1866589261$

1. 4600245058

$-0.2828136387$

0.088438048

0.6087872592

0.9175933331

$-0.0854526562$

0.3534312618

0.7637607203

0.6986369831

0.3146245896

1. 2157667625

$-0.0076505458$

$-0.7416450691$

- 1.7514405481

$-2.9367234828$

1. 5159390178

$-0.5782789649$

0.3287059322

1. 709538986 


$\begin{array}{lrrr}\mathrm{H} & -0.2260448495 & -1.754463724 & 1.7895787566 \\ \mathrm{H} & 0.7585092927 & -2.0555931758 & -1.1069461174 \\ \mathrm{H} & 2.1614203466 & -2.1446805634 & -0.0097201517 \\ \mathrm{H} & 1.5481964781 & 0.2898398728 & -2.7556614882 \\ \mathrm{H} & 2.7876228664 & -0.6114486491 & -3.660725181 \\ \mathrm{H} & 1.2044534805 & -1.3659550948 & -3.3348579043\end{array}$

